Gesnerus 55 (1998) 70-86

\title{
Mutation du concept de dégénérescence en Suisse romande 1870-1920*
}

Gilles Jeanmonod

\section{Summary}

Between 1870 and 1920 the concept of degeneration changed radically. Away from an organization principle of mental diseases, degeneration became progressively a disease entity capable of affecting the human species as a whole. In the French-speaking part of Switzerland, fear of a "degeneration of the race" reached considerable dimensions in the years around 1910. The study of the introduction and then diffusion of the degeneration concept reveals clearly the social commitment of the medical profession. Although the French-speaking Swiss psychiatrists showed no tendency to give clinical value to this concept, they are, howewer, at the origin, through this social discourse, of the success that the idea of degeneration had. Hygienist, antialcoholic and political environments took over the job to perpetuate this discourse and finally managed to empty the concept of degeneration of its substance.

\section{Résumé}

Entre 1870 et 1920, le contenu du concept de dégénérescence se modifie radicalement. De principe organisateur d'une conception globale des maladies mentales, cette notion se mue progressivement en entité morbide susceptible d'affecter l'espèce dans son ensemble. En Suisse romande, où la crainte de la «dégénérescence de la race» a pris une proportion considérable dans les

\footnotetext{
* L'auteur remercie vivement le docteur Jacques Gasser de l'Institut romand d'histoire de la médecine et de la santé pour ses précieux conseils et ses encouragements lors de la réalisation de cet article et le professeur François Jequier de l'Université de Lausanne, son directeur de mémoire.
} 
années 1910, l'étude de l'introduction puis de la diffusion du concept de dégénérescence est révélatrice de l'engagement social du corps médical. Ainsi, les psychiatres romands, peu enclins à accorder une quelconque valeur clinique à ce concept, sont cependant bien à l'origine du succès de l'idée de dégénérescence de l'espèce par leur discours social. Les milieux hygiénistes et antialcooliques, puis politiques se chargèrent ensuite de perpétuer ce discours et achevèrent de vider le concept de dégénérescence de sa substance.

A l'instar de ce qui se passe dans d'autres pays européens durant la même période, et de manière exemplaire en France, le concept de dégénérescence connaît en Suisse romande un remarquable glissement du discours psychiatrique au discours médical, puis à certains discours sociaux. Durant la cinquantaine d'années qui séparent la Guerre franco-allemande de la Première Guerre mondiale, la dégénérescence va en effet progressivement déserter les pages des ouvrages psychiatriques pour envahir celles de journaux de toute nature, au prix d'une définition toujours plus imprécise.

La notion de dégénérescence n'en était pas à ses premiers errements, sa définition variant alors suivant que l'on traitait du corps de l'individu ou du corps social. Ainsi, à partir du milieu du XVIII ${ }^{e}$ siècle, «dégénérer» caractérise pour Buffon le fait de dévier d'une espèce primitive, alors que pour Laënnec, au début du XIX ${ }^{\mathrm{e}}$ siècle, «dégénérescence» signifie transformation pathologique d'un tissu'. Avec Bénédict-Augustin $\mathrm{Morel}^{2}$, dans les années 1850, la dégénérescence acquiert une dimension anthropologique et psychiatrique. Puis, entre 1880 et 1900, c'est Valentin Magnan ${ }^{3}$ qui, en affinant la description des rapports entre processus dégénératif et nosologie psychiatrique et en fondant le concept sur un postulat évolutionniste, va réellement lancer l'idée de dégénérescence.

1 Sur les divers contenus du concept de dégénérescence, lire Anne KRAMS-LIFSCHITZ, «Dégénérescence et personne, migrations d'un concept au XIX ${ }^{e}$ siècle», in Biomédecine et devenir de la personne, sous la direction de S. Novaes, Paris, Seuil, 1991, pp. 131-158.

2 Cf. Bénédict-Augustin MOREL, Traité des dégénérescences physiques, intellectuelles et morales de l'espèce humaine, Paris, Baillière, 1857 et Traité des maladies mentales, Paris, Masson, 1860. Sur Morel et la théorie des dégénérescences, lire Ian DOWBIGGIN, La folie héréditaire ou comment la psychiatrie française s'est constituée en un corps de savoir et de pouvoir dans la seconde moitié du XIX $X^{e}$ siècle, traduit de l'américain par G. Le Gaufey (1991 pour l'édition originale), Paris, E.P.E.L., 1993.

3 L'ensemble de la conception de Magnan est exposé dans Valentin MAGNAN et Paul Maurice LEGRAIN, Les Dégénérés. Etat mental et syndromes épisodiques, Paris, Rueff, 1895. On peut lire en outre V. MAGNAN et P. SERIEUX, Le délire chronique à évolution systématique, Paris, Masson, 1892. Pour une biographie de Magnan, lire P. SERIEUX, Valentin Magnan: sa vie et son ceuvre, 1835-1916, Paris, Masson, 1918. 
Mais les beaux jours de la doctrine de Magnan seront de courte durée. De fait, si celle-ci est généralement acceptée comme facteur étiologique, l'énorme groupement nosologique qui en est issu ne convainc guère. Dès les années 1890, on se demande si la dégénérescence ne devient pas synonyme d'aliénation et si cette notion ne servirait pas à caser dans une classification pratique toutes les formes embarrassantes de la folie. A partir de 1900, son déclin s'accélère: l'essor de la neurologie et des psychothérapies va rapidement entraîner la disparition de l'idée de dégénérescence du discours psychiatrique.

Il n'en reste pas moins que cette théorie, cliniquement peu vérifiable, a doublement marqué l'histoire de la psychiatrie. D'une part, parce qu'elle constitue peut-être la première réalisation d'une explication globale des maladies mentales aboutissant à une nosologie certes critiquable, mais du moins cohérente. D'autre part, parce qu'elle a joué un rôle important, comme nous le verrons, dans la motivation de l'activité sociale des psychiatres et des médecins.

En Suisse romande, le début de la période qui nous intéresse est marqué par l'ouverture en 1873 de l'Asile de Cery près de Lausanne ${ }^{4}$. Cery et l'Asile des Vernets à Genève, relayé en 1900 par l'Asile de Bel-Air, constitueront les principaux centres psychiatriques de la région. A partir de 1876 à Genève et à partir de 1890 à Lausanne, la psychiatrie est d'ailleurs enseignée en faculté de médecine par les directeurs respectifs des deux asiles. Cette dimension universitaire des centres genevois et vaudois ne saurait cependant plonger dans l'oubli l'Asile de Préfargier près de Neuchâtel et l'Asile de Marsens dans le canton de Fribourg dont les médecins successifs ont souvent su faire autorité dans le domaine.

Entre 1890 et 1920, la notion de dégénérescence se répand dans la presse romande. Au point que dans les années 1910, elle est devenue centrale dans l'argumentation des milieux antialcoolique, hygiéniste, néo-malthusien et même politique, s'adaptant parfaitement dans le dernier cas à son inspiration fréquemment darwiniste sociale. Le beau succès d'une notion à laquelle bien peu alors se souciaient encore de donner une définition valable pose la question du rôle des psychiatres romands dans sa popularisation.

4 L'histoire administrative de l'Asile de Cery ainsi que les portraits de ses directeurs successifs sont exposés in Claude SECRETAN, L'Hôpital de Cery, 1873-1973, Lausanne, Payot, 1973. 


\section{Hérédité de la folie}

Avant de traiter du rôle des psychiatres dans cette affaire, il n'est peut-être pas inutile d'examiner rapidement quelles étaient les données qui cautionnaient la croyance en l'hérédité de la folie et pour commencer la notion de prédisposition ou de «diathèse». Dérivant de la tradition humorale, celle-ci signifie que «l'on n'héritait pas d'une pathologie particulière, mais d'un état prédisposant susceptible de rester latent ou de s'exprimer variablement d'un individu à l'autre par différents dysfonctionnements organiques» ${ }^{5}$. En médecine mentale, on dira alors que se transmet d'une génération à l'autre «un terrain propice, une prédisposition à la folie, plutôt qu'une lésion caractérisée de l'organe» ${ }^{6}$. Phénomène du reste fort rassurant pour les aliénistes, puisqu'il permet, comme le souligne Marc Jeannerod, de fournir «une justification scientifique à ce paradoxe de la maladie mentale de relever d'une cause à la fois $\left\langle\right.$ organique〉 et invisible» ${ }^{7}$. Dans ce système, une pathologie ne se déclare que lorsque sont conjugués dans l'organisme incriminé des facteurs intrinsèques, propres à cet organisme, et extrinsèques, issus du milieu. La maladie est alors le résultat de l'action de «causes prédisposantes» et de «causes déterminantes» ou «occasionnelles». En Suisse romande comme en France, la diathèse était connue depuis fort longtemps et demeurera très présente dans le discours médical jusque dans les années 1920.

Mais la notion de prédisposition n'était pas sans poser certains problèmes. La diathèse étant le résultat d'un processus héréditaire, on pouvait se demander comment elle pouvait survenir dans une lignée, ou s'il ne fallait pas remonter à la nuit des temps pour en découvrir l'origine. D'aucuns, à l'instar d'Auguste Chatelain, médecin en chef de la Maison de santé de Préfargier de 1872 à 1882, pensaient que la prédisposition à la folie pouvait être acquise puis, l'hérédité de l'acquis ne faisant aucun doute, transmise à la descendance. En 1889, l'aliéniste neuchâtelois donnait ainsi comme causes prédisposantes: la civilisation, décrite alors comme propice au surmenage du cerveau; le sexe, avec l'hystérie qui atteindrait la femme de préférence; l'âge, avec l'âge mûr considéré comme très propice à l'eclatement de folie; la profession, les professions entraînant le surmenage du cerveau se révélant en l'occurrence moins néfastes que celles qui amènent aux excès de boisson ou à l'exil et à un mal du pays délétère; et l'hérédité, la cause «de beaucoup

5 Pascale GLEIZE, «L'hérédité hors du champ scientifique», in Ethnologie française, 1994, no 1 , pp. 11-24; p. 13.

6 Marc JEANNEROD, De la physiologie mentale. Histoire des relations entre biologie et psychologie, Paris, Odile Jacob, 1996, p. 156.

7 Ibid., p. 156. 
la plus fréquente» ${ }^{8}$. Néanmoins, l'idée des prédispositions acquises devint source de confusion et de doute, car il est évident que certains facteurs se retrouvaient dans les deux ordres de causes.

A Genève, trois ans plus tard, Paul-Louis Ladame, médecin, neurologue, psychiatre et spécialiste d'anthropologie criminelle, mettait l'accent sur l'hérédité tout en reconnaissant pour sa part le rôle de «l'éducation vicieuse» dans la genèse de la prédisposition:

«La prédisposition à la folie vient de l'hérédité! Il y a une organisation cérébrale qu'on apporte en venant au monde et qui prédispose aux maladies nerveuses et mentales. (...). En réalité, il n'existe presque pas de cas d'aliénation mentale qui ne présente une origine héréditaire et pour lesquels on ne puisse trouver la cause prédisposante dans les générations précédentes. (...). Après l'hérédité, la cause prédisposante la plus redoutable des névroses et de l'aliénation, c'est la mauvaise éducation.»"

Toutefois, dès le début du $\mathrm{XX}^{\mathrm{e}}$ siècle, certains psychiatres romands ne se contentent plus d'invoquer la diathèse pour étayer leur conviction de l'hérédité des maladies mentales. De fait, les progrès de la recherche sur les mécanismes de l'hérédité, en particulier la redécouverte des lois de Mendel par Hugo de Vries et la réfutation de l'hérédité de l'acquis par August Weisman, donnaient à réfléchir sur une notion émanant d'une vieille tradition médicale. Auguste Forel sera le premier à proposer une solution de remplacement en Suisse romande. Estimant que «les maladies purement acquises du système nerveux ne peuvent comme telles créer de dispositions pathologiques héréditaires ${ }^{10}$, il développe la théorie de l'empoisonnement des germes séminaux ou «blastophorie».

«Sorte de «fausse hérédité», celle-ci «agit sur les germes non encore conjugués (...), et crée ainsi, à leur origine, ce qu'on appelle les 〈tares héréditaires〉 de toute nature, tandis que l'hérédité proprement dite ne fait que combiner et reproduire les énergies des ascendants.» ${ }^{11}$

L'influence de Forel sur Albert Mahaim, directeur de l'Asile de Cery de 1899 à 1925, fera de la blastophorie l'argument principal plaidant en faveur de l'évidence de l'hérédité des maladies mentales, et surtout de l'hérédité alcoolique, dans le milieu psychiatrique vaudois.

A Genève, par contre, certaines voix s'élèvent pour nuancer la toutepuissance de l'hérédité en psychiatrie. Le docteur Naegeli-Akerblom par exemple fustige dans un ouvrage publié en 1905 les «travaux médico-histo-

8 Auguste CHATELAIN, La folie. Causerie sur les troubles de l'esprit, Paris, Fischbacher, 1889, pp. 64-80.

9 Paul-Louis LADAME, «De l'hygiène morale», in Bibliothèque universelle et Revue suisse, 1892, t. LVI, pp. 106-124 et pp. 540-566; pp. 109-104.

10 Auguste FOREL, L'âme et le système nerveux, Paris, Steinheil, 1906, p. 199.

11 Auguste FOREL, La question sexuelle, Lausanne, Frankfurter, 1931, nouvelle édition (première édition, 1905), p. 48. 
riques au sujet de l'hérédité et de la dégénérescence» ${ }^{12}$, sapant ainsi un des piliers de l'a priori héréditaire dans le domaine. Neuf ans plus tard, Victor Demole, alors médecin assistant à l'Asile de Bel-Air, affirmait dans les fameuses Annales médico-psychologiques, s'appuyant sur un travail de Pearson, le directeur du Laboratoire d'Eugénique de Londres, qu'il convenait de s'interroger sérieusement sur la réalité de l'hérédité alcoolique ${ }^{13}$.

C'est ainsi que peu à peu la quasi unanimité qui s'était créée dans les deux dernières décennies du XIX ${ }^{\mathrm{e}}$ siècle autour de l'hérédité de la folie va se fissurer, laissant la place à des conceptions dans lesquelles l'approche organogénétique des maladies mentales sera moins dominante.

\section{Les psychiatres romands face à la notion de dégénérescence}

L'attitude que les psychiatres romands ont adoptée face à la doctrine des dégénérescences dépend de l'époque envisagée, de leurs options personnelles et des contextes dans lesquels ils s'expriment. En ce qui concerne l'époque, il est clair que le discours diffère suivant qu'il est émis lorsque le concept se trouve en plein développement, à son apogée ou sur son déclin. Sur les choix des psychiatres, il faut tenir compte d'une donnée importante, à savoir le décalage qui existe entre eux, essentiellement des praticiens, et une notion très théorique débouchant sur une nosologie cohérente mais cliniquement peu convainquante. Enfin, le contexte dans lequel ils s'expriment n'est pas indifférent, leurs préoccupations sociales les amenant souvent à utiliser une rhétorique dans laquelle la dégénérescence s'inscrit fort bien, en particulier dans la lutte antialcoolique et en psychiatrie légale.

\section{L'attitude mitigée des psychiatres romands}

De manière générale et à l'exclusion de certains contextes, les psychiatres sont demeurés en Suisse romande prudents et discrets au sujet de la notion de dégénérescence. Ce qui du reste pose problème à qui cherche à connaître leur opinion exacte sur la question, et il faut bien avouer que leur manque d'intérêt ou leur méconnaissance sont souvent les plus révélateurs.

12 Hans NAEGELI-AKERBLOM, Quelques résultats de l'examen des preuves historiques employées par les auteurs traitant de l'hérédité, Genève, Kündig, 1905, p. 80.

13 Victor DEMOLE, «Considérations biologiques sur l'hérédité dans les maladies mentales», in Annales médico-psychologiques, 1914, t. 5, pp. 417-431. 
La lecture de comptes-rendus de la Société des médecins aliénistes suisses, parus sporadiquement dans la Revue médicale de la Suisse romande entre 1884 et 1895, permet une évaluation sommaire au niveau suisse de l'importance de la dégénérescence. En effet, celle-ci n'y apparaît pratiquement pas, alors qu'elle atteint en France durant cette période l'apogée de sa carrière. En 1869 déjà, la même Société avait proposé une classification des maladies mentales qui ne contenait aucune allusion à la dégénérescence ${ }^{14}$. Peut-être faut-il interpréter ce désintérêt sur le plan suisse comme une conséquence de l'influence de la psychiatrie allemande, puisque chez nos voisins du nord la dégénérescence a relativement tôt été considérée comme une catégorie éventuelle de maladie mentale et non comme un concept global.

Voyons ce qu'il en est dans les cantons de Genève et de Vaud, traditionnellement plus tournés vers la France que vers l'Allemagne, et examinons les diverses nosologies qui sont présentées aux Vernets ou à Cery dans les rapports annuels.

Selon Philippe Rossignon, qui s'est penché sur l'histoire des classifications en vigueur à Genève, la «dégénérescence constitutionnelle» apparaît à l'Asile des Vernets en 1877 dans une nouvelle liste vraisemblablement établie par le docteur Olivet, médecin-directeur de cet établissement depuis $1856^{15}$. On ne peut en outre s'empêcher de relever la cö̈ncidence des dates entre la mise au point de cette nosologie et la nomination du docteur Olivet en 1876 comme professeur de psychiatrie à la faculté de médecine naissante. Quoiqu'il en soit, que la présence de la dégénérescence soit ici en partie imputable à une soudaine nécessité de perfectionnement théorique ou non, cette dénomination ne sera pas retenue longtemps aux Vernets.

A Lausanne, la lecture des Comptes-rendus du Département de l'intérieur du canton de Vaud de 1861 à 1901 et des Rapports annuels de la direction de Cery depuis 1901 semble indiquer un attrait pour la notion de dégénérescence légèrement plus grand et plus tardif qu'à Genève. Du docteur Zimmer, dernier médecin de l'Asile du Champ-de-l'Air, remplacé par Cery en 1873, jusqu'au docteur Mahaim, directeur de l'asile vaudois de 1899 à 1925, trois médecins-directeurs ont intégré la dégénérescence dans leurs classifications de 1891 à 1898. Il s'agit des docteurs Pachoud, Kohler et Rabow, et, nouvelle coïncidence peut-être, Frédéric Pachoud introduit cette notion dans son rapport un peu plus d'une année seulement après le début des cours

14 Cf. Charles LADAME, «Histoire de la Société des médecins-aliénistes suisses. 1864-1919», in Archives suisses de Neurologie et de Psychiatrie, 1922, pp. 130-141; p. 30.

15 Philippe ROSSIGNON, «L'évolution des classifications psychiatriques à Genève, du début du XIX ${ }^{e}$ siècle à aujourd'hui», in Revue médicale de la Suisse romande, 1996, no 11, pp. 861-870; p. 863. 
cliniques universitaires dispensés par Siegfried Rabow. Toutefois, dès 1899, Albert Mahaim renonce à recourir à la doctrine de Magnan, qui disparaît alors définitivement des rapports annuels de Cery.

Bien entendu, ces classifications restent des supports théoriques visant à étancher la soif statistique de l'administration et n'éclairent que partiellement le travail clinique des psychiatres. Par contre, les résultats de recherches effectuées dans les dossiers de patients par l'Institut romand d'histoire de la médecine et de la santé à Bel-Air et à Cery permettent bel et bien d'affirmer que les diagnostics de dégénérescence y sont fort rares entre 1870 et $1920^{16}$.

Comment expliquer un tel résultat en clinique, alors qu'en 1898, par exemple, le professeur Rabow déclare dans son rapport des taux de diagnostics de dégénérescence dans les entrées et les sorties atteignant respectivement $4,5 \%$ et $8,6 \%$ ? Une telle différence ne se comprend que si l'on tient compte des diverses exigences des deux types de discours envisagés, en l'occurrence le discours purement clinique et le discours plus théorique adopté lors de travaux administratifs.

Cette tendance à employer le concept de dégénérescence dans un cadre théorique se confirme du reste à l'examen de certains travaux parus dans les années 1890. C'est le cas dans un mémoire rédigé par Ernest Pronier, alors médecin-assistant à Cery, traitant de la contagion de la folie ${ }^{17}$. Dans cet ouvrage couronné par la Faculté de médecine de Genève, l'auteur estime par exemple que «parmi les délirants passifs, il en est beaucoup qui appartiennent à la classe des dégénérés, depuis le déséquilibré supérieur jusqu'à l'imbécile» ${ }^{18}$, se référant ainsi à Magnan. En outre, il conclut entre autres que la «réceptivité» agissant dans la contagion de la folie reconnât pour cause première «la dégénérescence psychique héréditaire» ${ }^{19}$. Cet exemple ne doit toutefois pas laisser croire que les travaux théoriques en psychiatrie parus en Suisse romande sont tous imprégnés de la notion de dégénérescence. La majorité de ceux que nous avons consultés ne traitent pas de celle-ci, du moins jusqu'à la fin du XIX ${ }^{\mathrm{e}}$ siècle.

16 Plus précisément, entre 1870 et 1920 sont diagnostiqués 3 cas de dégénérescence sur 839 hospitalisations ( $10 \%$ de toutes les hospitalisations) étudiées à Lausanne et 2 cas sur 527 hospitalisations ( $10 \%$ des hospitalisations également) étudiées à Genève. Ces données nous ont été communiquées par le docteur Jacques Gasser de l'Institut romand d'histoire de la médecine et de la santé, responsable du projet de recherche «Le passé dans le présent de la psychiatrie» qui fournit une analyse historique des dossiers de patients des asiles de Bel-Air et de Cery.

17 Ernest PRONIER, Etude sur la contagion de la folie, Lausanne, Pache, 1892.

18 Ibid., p. 20.

19 Ibid., p. 85. 
Aux environs de 1900, en effet, les références à la dégénérescence augmentent sensiblement dans la littérature psychiatrique; mais désormais il s'agit de manifestations de scepticisme. C'est le cas par exemple dans la seizième leçon des Psychonévroses et leur traitement moral de Paul Dubois qui, à la lecture d'un imposant tableau de Magnan regroupant les dégénérescences mentales, «craint que nous soyons tous des dégénérés» ${ }^{20}$. Dubois se méfie à vrai dire doublement de l'idée de dégénérescence, doutant d'une part de sa valeur clinique et estimant d'autre part qu'elle ne laisse guère d'espoir de «régénération», qu'elle décourage en fait la mise en œuvre de thérapies.

Pour Bernard Schwarzwald, auteur d'une thèse dirigée par le professeur Mahaim, ce n'est pas l'idée même de dégénérescence qu'il s'agit de mettre en question, mais le rôle que lui assignent Magnan et ses disciples ${ }^{21}$. Selon l'auteur, ceux-ci auraient en effet par trop invoqué l'hérédité des maladies mentales afin d'étendre l'emprise de la dégénérescence sur ces pathologies. En fait, si elle est bien susceptible de compliquer certaines psychoses, la dégénérescence n'en est pas pour autant la condition exclusive.

En définitive, nous retenons que l'attitude générale des psychiatres romands face à la notion de dégénérescence est des plus mitigée durant les décennies qui nous occupent. Cliniquement très peu utilisée, cette notion apparaît parfois dans des cadres théoriques ou administratifs, surtout dans les années 1880 et 1890, peut-être suite à la nécessité de mises au point théoriques induites par la création des facultés de médecine. A partir de 1900, tout comme en France, certains font part de leurs doutes; les belles années de la folie héréditaire et de la dégénérescence sont terminées.

Cette indifférence polie est imputable à notre sens à deux phénomènes. D'abord à une influence diffuse de la psychiatrie allemande, pour laquelle la dégénérescence apparaît comme une simple catégorie, ce que l'on retrouve dans les classifications concernées à Bel-Air et à Cery, et non comme un principe générateur fondamental. Ensuite à l'inadaptation du concept de dégénérescence, de nature théorique et totale, à l'activité de psychiatres tournés vers la clinique et plus familiers de prises de position théoriques ponctuelles que de conceptions globalisantes de la folie.

20 Paul DUBOIS, Les psychonévroses et leur traitement moral, Paris, Masson, 1909 (1904 pour la première édition), p. 232.

21 Bernard SCHWARZWALD, De la démence précoce familiale, Lausanne, Imprimerie cosmopolite, 1907. 


\section{Lutte antialcoolique et psychiatrie légale}

Si les psychiatres romands ont fait preuve de circonspection au sujet du concept de dégénérescence dans leur pratique et dans leur discours théorique, ils perdaient par contre fréquemment toute réserve lorsqu'ils traitaient d'alcoolisme ou de psychiatrie légale. La dégénérescence constituait en fait un élément majeur de l'argumentation élaborée autour de ces deux thèmes vitaux dans leur engagement social. Car les psychiatres ont effectivement joué un rôle important dans la lutte contre l'alcoolisme qui s'intensifiait en Suisse romande durant la deuxième moitié du XIX ${ }^{\mathrm{e}}$ siècle ${ }^{22}$, menant parallèlement campagne afin d'imposer leurs exigences en psychiatrie légale à partir des années $1880^{23}$.

En ce qui concerne la lutte antialcoolique, notre étude est axée sur le canton de Vaud et sur l'Asile de Cery. L'importance accordée par les psychiatres de 1890 à 1925 au rôle de l'alcool dans la genèse et le développement des maladies mentales y est de fait considérable.

Cette tradition remonte d'ailleurs presque aux premières années d'existence de l'asile ou, si l'on préfère, à son troisième médecin-directeur, le docteur Théodore Challand, en poste de 1876 à 1888. Ce Vaudois d'origine, docteur en médecine de la Faculté de Paris, a participé aux travaux de Magnan sur l'alcoolisme et publié une étude médicale sur le sujet en $1871^{24}$. Dix ans plus tard, il reprend les grandes lignes de son étude dans un court article qui aborde en sus certains aspects sociaux de l'alcoolisme ${ }^{25}$. De manière révélatrice, la dégénérescence, absente du premier travail, apparaît dans le second dans les préoccupations sociales de Challand surtout:

«Dans notre pays on constate, par exemple, que beaucoup d'aliénés qui sont soignés à l'asile de Cery sont atteints de dégénérescences héréditaires qui ne sont que le résultat de l'inconduite des parents et en particulier de leurs habitudes alcooliques. Quelquefois ce ne sont pas les descendants directs qui sont atteints, mais la génération suivante. Il n'est pas rare aussi de voir les descendants de buveurs avoir un goût marqué pour les boissons alcooliques et perpétuer ainsi cette tendance aux dégénérescences. (...). D'une manière générale on peut dire que l'alcoolisme est une des causes les plus actives de la dégénérescence de la race.»

22 Sur l'histoire de la lutte antialcoolique en Suisse romande, lire Elena GOTTRAUXBIANCARDI (présenté par), Air pur, eau claire, préservatif. Tuberculose, alcoolisme, sida: une histoire comparée de la prévention, Lausanne, Editions d'en bas, 1992.

23 Sur les enjeux de la psychiatrie légale en Suisse romande à la fin du XIX ${ }^{e}$ siècle, lire Vincent BARRAS et Jacques GASSER, «LLe drame de Brenles». Fondements de la psychiatrie légale en Suisse romande», in Revue médicale de la Suisse romande, 1996, no 11, pp. 901-907.

24 Théodore CHALLAND, Etude expérimentale et clinique sur l'absinthisme et l'alcoolisme, Paris, Delahaye, 1871.

25 Théodore CHALLAND, De l'alcoolisme et des moyens de le combattre, extrait du Journal de la Société vaudoise d'Utilité publique, Lausanne, Bridel, 1881. 
On trouve ainsi en 1881 déjà dans cet article ${ }^{26}$ d'un directeur de Cery tous les éléments qui caractériseront le discours antialcoolique des vingt premières années du $\mathrm{XX}^{\mathrm{e}}$ siècle: assimilation de l'alcoolisme à une maladie mentale, hérédité de l'alcoolisme et processus dégénératif menaçant la race. Chez Challand, qui utilise dans ses rapports des classifications ne mentionnant pas la notion de dégénérescence, celle-ci est déjà en train de glisser des «dégénérescences», concept anthropologique et psychiatrique, à la «dégénérescence de la race», concept socio-biologique.

La même ambiguïté apparaît chez Siegfried Rabow. En 1890, il affirme, en reprenant à son compte une citation anonyme, que «l'usage excessif de spiritueux de toute nature est une des causes principales de la dégénérescence physique, intellectuelle et morale des nations comme des individus» ${ }^{27}$; et six ans plus tard, la dégénérescence apparaît pourtant dans sa classification des maladies mentales.

Après cette période de transition, qui voit se côtoyer dans le discours psychiatrique le concept médical et le concept social antialcoolique de la dégénérescence, se met en place avec Albert Mahaim une nouvelle approche qui se contente de l'emploi social de la notion. Ce qui signifie que celle-ci est désormais destinée à renforcer le discours vulgarisateur et public sur l'hérédité alcoolique et les maladies mentales. La blastophorie, censée remplacer la diathèse, se voit alors à son tour associée à la dégénérescence: «les dégénérescences alcooliques de la race (...) sont le fait de la seule blastophorie», affirme Forel ${ }^{28}$.

Mais vers la fin des années 1910, les psychiatres vaudois, toujours autant impliqués dans cette lutte, se sont rendu compte depuis quelques années que la dégénérescence nuisait à la cause, et ceci même dans son emploi social. C'est que le scénario d'extinction des lignées issu de Morel et Magnan était resté suffisamment vivace dans les esprits pour incliner certains à penser qu'il était vain de lutter contre l'alcoolisme. Les psychiatres, se voyant en quelque sorte trahis par leur propre créature, allaient se distancier à partir de 1920 de l'idée de dégénérescence.

En médecine légale, les psychiatres romands ont également mis en place un discours théorique et public au cours des vingt dernières années du XIX ${ }^{\mathrm{e}}$ siècle. Ceci prend place du reste dans le grand débat qui oppose en Europe les défenseurs de l'Ecole positiviste italienne d'une part et la majorité des

26 Ibid., pp. 73-74.

27 Siegfried RABOW, «Leçon d'ouverture de psychiatrie», in Discours et leçons prononcés à l'ouverture des cours du premier semestre de l'Université de Lausanne, octobre 1890, Lausanne, 1891, p. 237.

28 Auguste FOREL, La question sexuelle, op. cit. (supra n. 11), pp. 48-49. 
magistrats et juristes d'autre part, et qui voit niée par les premiers la notion de libre arbitre, chère aux seconds, au profit de l'idée de responsabilité sociale des criminels, chère à Lombroso.

Afin d'avoir leur mot à dire sur la question du crime, il est bien clair que les psychiatres se doivent comme pour l'alcoolisme d'affirmer sa morbidité, dans certains cas au moins. C'est dans ce contexte que s'impose tout naturellement la notion de dégénérescence déjà fort connue par l'intermédiaire de la lutte antialcoolique, susceptible de convaincre quelques magistrats et propre à frapper l'opinion publique. L'amalgame dégénérescence-alcoolcrime apparaît par exemple à Cery en 1892:

«Outre que l'alcoolisme est une cause fréquente de crime, il constitue pour les descendants une forte tare héréditaire qui se traduit par les stigmates physiques et moraux des dégénérés, prédisposés à tous les mauvais penchants, et qui fournissent un si grand contingent à l'asile et à la prison. ${ }^{29}$

L'affirmation de la morbidité du crime ne constitue cependant que la base du programme socio-criminel des psychiatres. Afin de protéger la société des malades mentaux aliénés considérés comme irresponsables, et parfois incurables, ils estiment en effet nécessaire de pouvoir interner ceux-ci pour une durée illimitée. Deux mesures concrètes accompagnent cette exigence: la mise en place d'une pénalité qui ne lie pas la peine à la faute, mais au criminel et à sa dangerosité; et la création d'établissements propres à accueillir des malades dangereux.

Dans ces prétentions qui tournent à la propagande au début du $X X^{\mathrm{e}}$ siècle, il n'est donc pas étonnant de retrouver la dégénérescence aussi bien dans des thèses ou des articles médico-légaux que dans les chroniques judiciaires. L'irresponsabilité, que l'on considère volontiers à l'exemple de Forel comme en partie fonction de la gravité des atteintes cérébrales ${ }^{30}$, et l'incurabilité, justifiée par l'idée de déterminisme biologique, s'associent de fait très bien à un concept imprégné d'organicisme et d'omnipotence de l'hérédité. Les résultats de cette campagne ne se font pas attendre. Dès 1900, des criminels présentés comme aliénés et alcooliques sont déclarés irresponsables en partie ou totalement, acquittés puis internés administrativement. Et en 1906 est adoptée dans le canton de Vaud la «loi sur l'internement des alcooliques» inspirée des thèses psychiatriques ${ }^{31}$.

29 Ed. CHARBONNIER, Remarques sur quelques cas de meurtres commis par des aliénés d'après des observations faites à l'Asile de Cery, Lausanne, Borgeaud, 1892.

30 Auguste FOREL et Albert MAHAIM, Crime et anomalies mentales constitutionnelles, Genève, Kündig, 1902, pp. 7-12.

31 Pour plus de données sur l'emploi de la notion de dégénérescence en psychiatrie légale, lire Gilles JEANMONOD, Approche de l'histoire de l'hérédité en Suisse romande: de quelques emplois de la notion de dégénérescence, mémoire de licence, Lausanne, 1996, pp. 62-69. 
Finalement, c'est bien l'importance accordée à leur engagement social, autrement dit leur volonté d'affirmer le fonctionnement de la psychiatrie comme «forme d'hygiène publique» ${ }^{32}$, qui a amené les psychiatres à employer une notion dont ils se désintéressaient en d'autres circonstances. Si cette attitude dénote une certaine duplicité, elle indique aussi que la notion de dégénérescence était peut-être mieux adaptée à certains discours sociopolitiques qu'à la psychiatrie.

\section{La notion de dégénérescence dans le discours médical}

Si les psychiatres semblent bien avoir joué un rôle décisif dans la diffusion de l'idée de dégénérescence dans son acception sociale, il n'en ont cependant pas été les uniques acteurs. Les médecins généralistes avaient très tôt compris également le parti à tirer de ce concept. Engagés dans le courant hygiéniste au sens large, leurs motivations étaient en fait identiques à celles des psychiatres qui exaltaient pour leur part plus particulièrement l'hygiène mentale. Une des premières entités morbides associée par les médecins à la dégénérescence est sans doute le crétinisme qui avait du reste fort préoccupé Morel lui-même. Spécialiste de la question, le docteur Lombard de Genève affirmait en 1873:

«L'endémie du crétinisme co-existe toujours avec celle du goître et avec certaines dégénérescences qui annoncent un abaissement de la vitalité; ce sont: la surdimutité, le rachitisme, l'imbécilité et l'idiotie ainsi que les affections scrofuleuses et tuberculeuses. Comme on le voit, le goître et le crétinisme ne sont que deux degrés de la dégénérescence de l'espèce humaine $(\ldots) . »^{33}$

Près de dix ans avant Challand à propos de l'alcoolisme, le même glissement sémantique s'opère ici des dégénérescences affligeant l'espèce humaine (c'est-à-dire seulement certains éléments de cette espèce, une famille, une lignée) à la dégénérescence menaçant l'espèce dans son entier. A l'instar des psychiatres, les médecins ont bien usé par la suite de la notion de dégénérescence dans ses acceptions première et dérivée, mais de manière sensiblement différente.

Il semble par exemple qu'ils se soient relativement peu servis de l'argument de la dégénérescence dans leur participation à l'effort de lutte contre

32 Michel FOUCAULT, «Evolution de la notion d'individu dangereux» dans la psychiatrie légale», in Déviance et Société, 1981, no 4, pp. 403-422; pp. 408-409.

33 H.-C. LOMBARD, «Etude sur le goître et le crétinisme endémique et sur leur cause atmosphérique, lue à la Société médicale de Genève le 5 novembre 1873», in Bulletin de la Société médicale de la Suisse romande, 1874, pp. 6-24 et pp. 55-63; p. 9. 
l'alcoolisme. Une enquête menée en 1895 par une société antialcoolique montre que seuls deux médecins sur quatorze mentionnent des risques de dégénérescence au sens psychiatrique, et qu'aucun n'invoque la dégénérescence de la race ${ }^{34}$. Plus tard, lorsque paraîtront des revues entièrement consacrées à l'hygiène, il est vrai que des médecins reprendront fréquemment ce thème qui avait alors largement pénétré le discours sociopolitique.

Mais il n'est pas rare que des généralistes versés dans le domaine psychiatrique accordent un certain crédit à la théorie de Magnan dans des textes plus spécialisés. Paul-Louis Ladame, médecin dont les vastes connaissances «neuro-psychiatriques» ne lui permettront cependant jamais d'exercer la psychiatrie dans un cadre universitaire, en est un bon exemple. De 1880 à 1910, il écrit quelques articles desquels il ressort une reconnaissance implicite de la théorie des dégénérescences. Dans une de ses observations datant de 1889, l'anamnèse du patient nous apprend que celui-ci est un «menteur effronté, dès son bas âge, gourmand, paresseux, voleur, colère, comme la plupart des héréditaires dégénérés ${ }^{35}$. Quatre ans plus tard, le même Ladame assure que «l'obsession du meurtre appartient à la grande classe de la folie héréditaire» et qu'elle «se présente habituellement sous la forme d'un 〈syndrome épisodique〉 (Magnan) de la dégénérescence mentale» ${ }^{36}$.

Néanmoins, s'il est vrai que, dans l'emploi de la dégénérescence, la personnalité du médecin compte, certains thèmes également semblent propices à son apparition, comme la «neurasthénie» ou la «psychasthénie». Jean Conelli écrit ainsi dans La neurasthénie que «tous les états névropathiques sont transmissibles par l'hérédité et s'aggravent dans cette transmission en aboutissant au phénomène final inévitable de la dégénérescence» ${ }^{37}$, alors que le docteur Castanié n'hésite pas quant à lui à diagnostiquer une «psychasthénie dégénérative» ${ }^{38}$.

Des psychologues participent du reste aussi de cette tendance sanctionnant la validité de la dégénérescence. A l'exemple de Edouard Claparède

34 COMITÉ CENTRAL DE L'ESPOIR, Préservons nos enfants ou l'enfance et les boissons enivrantes, Rolle, Secrétariat général de L'Espoir, ca. 1905.

35 Paul-Louis LADAME, «De l'épilepsie procursive», in Revue médicale de la Suisse romande, 1889, pp. 5-21; p. 6.

36 Paul-Louis LADAME, «L'obsession du meurtre», in Actes du troisième Congrès international d'anthropologie criminelle tenu à Bruxelles en août 1892, Bruxelles, Hayez, 1893, pp. 41-55.

37 Jean Anténor CONELLI, La neurasthénie, thèse de doctorat de la Faculté de Médecine de Lausanne, Turin, Roux Frassati, 1895, p. 33.

38 Adrien CASTANIE, «L'influence de la rééducation dans un ças grave de psychasthénie», in Revue suisse de Médecine, 1909, t. 1, pp. 80-87; p. 85. 
qui traite d'une «dégénérescence d'évolution ${ }^{39}$ ou de Auguste Lemaître qui discute du cas d'un «dégénéré supérieur» ${ }^{40}$.

Ces emplois médicaux de la notion de dégénérescence s'expliquent si l'on tient compte de certaine volonté des médecins, des psychologues et des neurologues à l'aube du $\mathrm{XX}^{\mathrm{e}}$ siècle qui affirme la nécessité de traiter spécifiquement une partie des affections mentales dont les psychiatres ne s'occupaient guère. Selon Paul Dubois, les états psychopathiques que recouvrent les psychonévroses, en particulier l'hystérie et la psychasthénie, sont compatibles avec la vie dans la famille, dans la société; ils ne nécessitent pas l'internement et le malade peut fort bien s'adresser alors à son médecin ou aux «neurologistes» ${ }^{41}$. Cette volonté des médecins de s'affirmer en médecine mentale les pousse ainsi à s'enquérir de données théoriques dont ils n'ont pas l'occasion de vérifier le bien-fondé clinique. Et c'est tout à fait naturellement que la littérature psychiatrique française les amène à des diagnostics impliquant la notion de dégénérescence.

\section{Glissement de la notion de dégénérescence dans le discours socio-politique}

Exploité à des fins sociales par les psychiatres, parfois mal compris par des médecins qui l'accommodent un peu à leur gré, vite récupéré par les mouvements antialcooliques et hygiénistes, le concept de dégénérescence va terminer sa course entièrement galvaudé dans le discours socio-politique ${ }^{42}$.

Rappelons que ces cinquante années de carrière sont contemporaines de l'émergence d'une réelle volonté de biologisation des mécanismes sociaux. Très rapidement, les théoriciens du darwinisme social avaient compris que l'idée de dégénérescence de la race illustrait parfaitement la marche d'une société privée de la sélection naturelle, c'est-à-dire un mouvement opposé au progrès. Plus encore, selon Daniel Pick, la hantise de la dégénérescence qui s'empare de la France, de l'Angleterre ou de l'Italie dans le dernier tiers du $\mathrm{XIX}^{\mathrm{e}}$ siècle est caractéristique d'entités nationales plongées dans le doute

39 Edouard CLAPARÈDE, «L'illusion de poids chez les anormaux et le «signe de Demoor»», in Archives de Psychologie de la Suisse romande, 1903, t. 2, pp. 22-32; p. 32.

40 Auguste LEMAÎTRE, «Contribution à la psychologie de l'adolescent. - III. L'évolution mentale d'un dégénéré supérieur», in Archives de Psychologie de la Suisse romande, 1909, t. 8, pp. 241-262.

41 Paul DUBOIS, op. cit. (supra n. 20), pp. 18-19.

42 Pour plus de précisions sur les divers aspects des emplois socio-politiques de la notion de dégénérescence, lire Gilles JEANMONOD, op. cit. (supra n. 31), pp. 81-90. 
quant à leur avenir social, politique et économique ${ }^{43}$. Et il apparaît que la Suisse romande au début du $\mathrm{XX}^{\mathrm{e}}$ siècle connaît semblable période de crise, si l'on pense aux grèves survenues à Genève et dans le canton de Vaud en 1902 et en 1907 ou aux peurs éprouvées face à l'essor du socialisme.

Il faut comprendre dans ces conditions que de constantes références à la dégénérescence n'occupent pas uniquement les pages d'organes antialcooliques ou hygiénistes, dans lesquels cette présence n'a rien d'étonnant, mais également celles de journaux marqués par une tendance politique ou confessionnelle qui se montrent généreux en images frappantes évoquant les ravages de la dégénérescence.

Les causes de la dégénérescence qui sont le plus fréquemment invoquées dans ces feuilles politiques rappellent celles énoncées par Morel. Les causes relevant du milieu social mettent en évidence la misère du prolétariat, le travail en usine, le travail des enfants, les logements insalubres, la vie urbaine, mais aussi l'excès de travail intellectuel et le sédantarisme. Certaines affections morbides comme la tuberculose ou la syphilis figurent bien entendu parmi les causes. Enfin, l'intoxication par l'alcoolisme représente la menace suprême, souvent associée à la criminalité.

Si l'idée de dégénérescence de la race apparaît dans les journaux au début des années 1890, ce n'est qu'à partir des années 1905 à 1910, période d'intenses campagnes antiabsinthiques, qu'elle envahit certains discours politiquement orientés, y demeurant très présente pendant une quinzaine d'années. Il est assez délicat d'aborder cet aspect de l'histoire de la dégénérescence, celle-ci étant considérée depuis l'émergence du nazisme comme une entité caractéristique d'idéologies d'extrême-droite. Mais la lecture de la presse politique des années concernées ne laisse guère de doutes: les journaux de gauche ont largement usé de l'argument de la dégénérescence. Si la droite, dans le sillage du darwinisme social, l'utilisait pour justifier les inégalités sociales, la gauche s'en servait en particulier pour fustiger les politiques natalistes et l'alcoolisme, présentés comme des moyens d'asservir le prolétariat. Précisons que cette constatation n'a absolument rien de commun avec certaines menées de la Nouvelle droite française cherchant à banaliser l'idéologie d'extrême-droite en soulignant des aspects du discours de la gauche des années 1900 à $1920^{44}$.

43 Daniel PICK, Faces of degeneration. A european disorder, c. 1848-c. 1918, Cambridge, Cambridge University Press, 1989.

44 Une telle tentative a vu le jour par exemple en 1982, année du centenaire de la mort de Darwin, dans la revue Nouvelle Ecole, no 38. On y tente de cautionner l'actuelle «sociobiologie» en mettant en évidence l'inspiration darwiniste sociale d'une poignée de penseurs de gauche. 
Très loin de cette rhétorique dangereuse, nous conclurons que si le thème de la dégénérescence de la race a pénétré aussi bien le discours des élites bourgeoises que celui de la gauche socialiste et syndicaliste, c'est qu'il était bien un thème porteur. Ainsi la peur collective qu'engendrait cette menace diffuse permettait de resserrer les rangs des partisans de tous bords.

Pour répondre à la question initiale du rôle des psychiatres dans la popularisation de la notion de dégénérescence, nous dirons qu'ils en sont sans doute à l'origine et qu'ils y ont bien contribué par leur discours social exclusivement, étant admise leur méconnaissance de la question au niveau clinique, glissant rapidement à l'idée de dégénérescence de la race. Les mouvements hygiénistes et antialcooliques se chargeant ensuite de diffuser plus largement l'annonce du danger encouru par l'espèce, ceci d'autant plus aisément que le darwinisme social recevait des échos favorables.

Dans le fond, si l'histoire de la notion de dégénérescence en Suisse romande nous apprend peu de chose sur la pratique psychiatrique elle-même, elle n'en reste pas moins très utile pour comprendre l'extrême importance qu'attribuaient les psychiatres et les médecins à leur mission sociale en ce début de XX ${ }^{\mathrm{e}}$ siècle. Car ainsi que l'affirme le docteur Messerli en mai 1917 dans la Gazette d'Hygiène et de Médecine de la Suisse romande, «le médecin moderne ne peut plus être considéré uniquement comme un thérapeute; sa position sociale veut qu'il soit aussi un hygiéniste, un pédagogue, un psychologue, etc., en un mot un vrai sociologue; il se doit non seulement à ses malades, mais à toute la société, à tous ses contemporains». 